\title{
Percepções sobre o preceptor cirurgião-dentista da Atenção Primária à Saúde no ensino da Odontologia
}

\author{
Gabriela Walter da Luz*; Ramona Fernanda Ceriotti Toassi**
}

* Pós-graduanda, Residência Integrada em
Odontologia da Faculdade de Odontologia,
Universidade Federal do Rio Grande do Sul
** Professora Doutora do Departamento de Odontologia
Preventiva e Social da Faculdade de Odontologia e
do Programa de Pós-graduação em Ensino na Saúde,
Universidade Federal do Rio Grande do Sul

\section{RESUMO}

A implantação das Diretrizes Curriculares Nacionais para os cursos da área da saúde no Brasil, incluindo a Odontologia, trouxe a necessidade da integração do ensino com o Sistema Único de Saúde. Essa integração é possibilitada pela experiência dos estudantes nos estágios curriculares junto aos serviços públicos de saúde, acompanhados pelo preceptor, profissional que atua no serviço. $\mathrm{O}$ objetivo desta pesquisa foi compreender a percepção dos cirurgiões-dentistas, professores supervisores e estudantes sobre os preceptores do estágio curricular supervisionado da Odontologia na Atenção Primária à Saúde (APS). Trata-se de um estudo exploratório qualitativo realizado por meio de entrevistas semiestruturadas com estudantes, professores e preceptores $(n=20)$ vinculados ao estágio curricular do curso de Odontologia da Universidade Federal do Rio Grande do Sul nos serviços de APS. O preceptor cirurgiãodentista foi percebido como o profissional que insere e integra o estudante à equipe de saúde e à rotina do serviço durante o estágio. Quando demonstrou receptividade ao estudante, competência didático-pedagógica e segurança no trabalho, atuou como facilitador da aprendizagem. Experiências positivas de preceptoria, junto a um profissional considerado 'competente', resultaram em um modelo de trabalhador e colega para os estudantes. A relação do estudante com o preceptor não foi percebida como a que o estudante tem com o professor na universidade. A aproximação da universidade ao preceptor do estágio, apoiando-o nas atividades que desenvolve com os estudantes no cotidiano do trabalho deve ser entendida como condição necessária para a integração ensino-serviço-comunidade.

Descritores: Educação em Odontologia. Atenção Primária à Saúde. Serviços de Saúde. Preceptor. Pesquisa Qualitativa.

\section{INTRODUÇÃO}

Historicamente, o ensino da Odontologia no Brasil se constituiu tendo um enfoque excessivo na abordagem técnico-científica, fragmentando os conteúdos e formando profissionais voltados para a especialização com um precário conhecimento da realidade das condições de saúde da população ${ }^{1,2}$.

O movimento de mudanças para uma formação adequada à realidade local do país passou a se constituir uma preocupação 
constante dos currículos de cursos da área da saúde, tanto no Brasil quanto no contexto internacional. Para os cursos de Odontologia, as Diretrizes Curriculares Nacionais $(\mathrm{DCN})^{3}$ foram aprovadas mais recentemente e vieram regulamentar e apontar a necessidade de formação para o sistema de saúde vigente no país.

As DCN significaram um avanço dos projetos pedagógicos dos cursos de graduação, estabelecendo competências e habilidades para a formação de um cirurgiãodentista generalista, com visão mais humanizada, política e voltada para o cuidado integral à saúde da população e garantiram o desenvolvimento dos estágios curriculares de forma articulada com complexidade crescente ao longo do processo de formação do cirurgião-dentista e com supervisão docente ${ }^{4}$.

Esse novo cenário trouxe a necessidade da integração do ensino com o Sistema Único de Saúde (SUS), possibilitando ao estudante a articulação do conhecimento técnico adquirido à prática dos serviços e realidade das comunidades ${ }^{5}$.

Na Atenção Primária à Saúde (APS), orientadora do cuidado nas redes de atenção à saúde, o estágio curricular tem potencial para possibilitar uma vivência concreta da realidade que o estudante encontrará no país durante o exercício profissional, após a conclusão do curso de graduação ${ }^{6}$. São atores dessa integração juntamente com os professores (supervisores), estudantes e a comunidade, os preceptores, profissionais da saúde com função de supervisão docente-assistencial por área específica de atuação ou de especialidade profissional, que exercem atividade de organização do processo de aprendizagem especializado e de orientação técnica aos estudantes em estágio ou em vivências da graduação ${ }^{7}$. Ao professor cabe a supervisão de um grupo maior de estudantes nos cenários de prática dos estágios, sendo capaz de apoiar, aconselhar, instruir e problematizar as experiências vividas, facilitando o processo de aprendizado significativo, além de avaliar o aprendizado dos estudantes e o próprio cenário de prática ${ }^{6,8}$.

$\mathrm{Na}$ Universidade Federal do Rio Grande do Sul (UFRGS), o curso de graduação em Odontologia, prevendo a formação de profissionais voltados para o atendimento humanizado e em acordo com os princípios do SUS, reestruturou seu currículo baseando-se nas $\mathrm{DCN}^{3}$. O currículo implantado a partir de $2005^{9}$ passou a contemplar a atuação do cirurgião-dentista enquanto agente modificador de saúde, portador de visão crítica do espaço de sua realidade e com uma sólida formação técnico-científica e humanística. Para tanto, a ênfase na integração ensinoserviço-comunidade por meio de estágios curriculares supervisionados no SUS foi uma das grandes inovações curriculares. Esses estágios acontecem no último ano da graduação, sendo o do $9^{\circ}$ semestre nos serviços de APS, e o do $10^{\circ}$ semestre nos serviços de média e alta complexidade e gestão em saúde pública.

Pesquisa de abordagem qualitativa realizada no curso de Odontologia da UFRGS - estágio curricular nos serviços de APS mostrou a potencialidade desse estágio na formação do cirurgião-dentista para o estabelecimento de vínculos, competência da autonomia na resolução de problemas e trabalho em equipe multiprofissional. Foram evidenciadas aprendizagens sobre funcionamento dos serviços de saúde, cuidado em saúde e desenvolvimento de competência cultural ${ }^{10}$.

Nesse contexto de ensino nos serviços de saúde, o preceptor é o profissional não 
pertencente ao corpo docente da Instituição de Ensino Superior que tem o papel de suporte ao estudante na sua inserção no estágio curricular. O preceptor deve atuar como um facilitador da aprendizagem do estudante por meio de uma organização do processo de trabalho que comporte o ensino e o compartilhamento de experiências de forma que seja possível a integração de conceitos adquiridos na universidade com os do serviço $^{11}$.

Entendendo a importância do preceptor para criar condições necessárias para as mudanças pretendidas na formação em saúde, esta pesquisa propôs-se a compreender a percepção dos cirurgiões-dentistas, professores supervisores e estudantes sobre os preceptores do estágio curricular supervisionado da Odontologia na APS.

\section{METODOLOGIA}

Trata-se de estudo exploratório de base qualitativa, cujo objeto de investigação foi o preceptor cirurgião-dentista do estágio curricular supervisionado do curso de Odontologia da UFRGS nos serviços de APS de Porto Alegre, Rio Grande do Sul.

A metodologia seguiu os critérios para pesquisa qualitativa recomendados por Tong, Sainsbury e Craig ${ }^{12}$.

A coleta de dados aconteceu em 2011 por meio da realização de entrevistas semiestruturadas. Estudantes, professores e preceptores participaram das entrevistas, após a assinatura do Termo de Consentimento Livre e Esclarecido. Os grupos foram entrevistados de acordo com suas disponibilidades de tempo ao longo de quatro meses.

As entrevistas foram realizadas face-aface, por uma única pesquisadora, professora na UFRGS, com título de doutorado e experiência prévia com pesquisas qualitativas.

Foram realizadas no espaço da Faculdade de Odontologia e, com alguns dos preceptores, em seus locais de trabalho ou também na Faculdade de Odontologia. Todas as entrevistas seguiram um roteiro prétestado, foram individuais, gravadas em equipamento de áudio e integralmente transcritas.

Todas as transcrições foram devolvidas aos entrevistados para que pudessem lê-las, verificando se estavam de acordo com as ideias apresentadas e, se julgassem necessário, complementassem seus relatos. Nenhuma entrevista teve que ser repetida e nenhum participante abandonou o estudo. A duração das entrevistas variou de 45 minutos à uma hora e trinta minutos.

Os sujeitos dessa pesquisa foram escolhidos de modo intencional - amostra intencional - levando em consideração o objetivo proposto. $\mathrm{O}$ critério para inclusão dos estudantes foi terem concluído o Estágio Curricular Supervisionado na APS/UFRGS, ou seja, estarem no último semestre da graduação $\left(10^{\circ}\right.$ semestre). Os professores e preceptores deveriam estar atuando no estágio pelo período mínimo de um ano.

O método de amostragem utilizado foi o da saturação ${ }^{13}$, ou seja, quando se entendeu que novas falas passaram a ter acréscimos pouco significativos em vista dos objetivos propostos pela pesquisa e tornaram-se repetitivas, a coleta de dados foi encerrada.

Ao final, 20 indivíduos participaram do estudo, sendo 11 estudantes da graduação em Odontologia, 3 professores do estágio curricular supervisionado e 6 preceptores cirurgiões-dentistas dos diferentes serviços de APS de Porto Alegre (tabela 1). 
Tabela 1 - Distribuição dos participantes do estudo segundo as variáveis sexo, idade e tempo de preceptoria

\begin{tabular}{|c|c|c|c|}
\hline VARIÁVEIS & ESTUDANTES & PRECEPTORES & PROFESSORES \\
\hline \multicolumn{4}{|l|}{ Sexo } \\
\hline Feminino & 6 & 2 & 2 \\
\hline Masculino & 5 & 4 & 1 \\
\hline \multicolumn{4}{|l|}{ Idade (anos) } \\
\hline $21-22$ & 4 & -- & -- \\
\hline $23-25$ & 7 & -- & -- \\
\hline $29-30$ & -- & 3 & 1 \\
\hline $31-34$ & -- & 1 & -- \\
\hline $35-37$ & -- & -- & 1 \\
\hline $42-48$ & -- & 2 & 1 \\
\hline Tempo de preceptoria & -- & & -- \\
\hline 1 ano e meio ( 3 semestres) & -- & 1 & -- \\
\hline 2 anos (4 semestres) & -- & 2 & -- \\
\hline 3 anos (6 semestres) & -- & 1 & -- \\
\hline 3 anos e meio ( 7 semestres) & -- & 2 & -- \\
\hline TOTAL & 11 & 6 & 3 \\
\hline
\end{tabular}

A análise do material verbal obtido seguiu o método da análise de conteúdo temática de Bardin $^{14}$, com o auxílio do software para análise de dados qualitativos ATLAS.ti ${ }^{\circledR}$ (Visual Qualitative Data Analysis, Berlin, Germany). A utilização do software facilitou a organização do material textual em categorias de análise (categorias emergentes foram constituídas ao se agrupar os temas mais frequentemente relatados nas falas dos sujeitos da pesquisa).

As categorias foram estabelecidas por ambos pesquisadores a partir do conteúdo das entrevistas - categorias emermegente - , não sendo estabelecidas previamente.

O estudo foi aprovado pelo Comitê de Ética em Pesquisa da Universidade Federal do Rio Grande do Sul (protocolo 18139).

\section{RESULTADOS E DISCUSSÃO}

Os estágios curriculares têm se incorporado aos currículos dos cursos de graduação da área da saúde, com a intenção de possibilitar aos estudantes vivências em campos de trabalho fora da universidade, para que possam conhecer a profissão inserida em sua realidade ${ }^{15}$. Tais experiências curriculares buscam melhorar a formação, promover engajamento social e conhecer as necessidades da população, colocando o estudante em contato com as práticas e políticas em saúde pública e ampliando as relações ensino-serviço ${ }^{16}$.

O ensino no serviço constitui, assim, 
uma estratégia pedagógica de integração da universidade aos serviços de saúde pública. $\mathrm{O}$ conteúdo programático ensinado aos estudantes é elaborado de acordo com a realidade do serviço para que se possa estabelecer a conexão da teoria e da prática. Essa integração gera maior entendimento da realidade da comunidade e possibilita a identificação de prioridades no cuidado em saúde ${ }^{2,17}$.

Ao inserir o estudante no serviço, ele passa a trabalhar juntamente com o cirurgiãodentista trabalhador do SUS em uma equipe multiprofissional, buscando atuar de forma interdisciplinar. Esse cirurgião-dentista assume o papel de preceptor do estudante de graduação. O preceptor é o profissional que não pertence ao corpo docente e que é responsável pela inserção e socialização do estudante no ambiente de trabalho, estreitando, assim, a distância entre teoria e trabalho ${ }^{18}$.

Os resultados estão apresentados em três categorias (unidades de significação) que emergiram das falas dos estudantes, professores e preceptores: a) chegada do estudante ao serviço: a receptividade do preceptor e o vínculo com a equipe; b) preceptorprofessor: a importância da competência didático-pedagógica; e c) integração teóricoprática: o preceptor enquanto facilitador da integração dos conhecimentos teóricos com a realidade do serviço.

\section{Chegada do estudante ao serviço: a receptividade do preceptor e o vínculo com a equipe}

Ao chegarem ao serviço de APS, os estudantes reconhecem o preceptor como um agente de integração entre eles e a equipe de saúde multiprofissional que atua nas Unidades de Saúde. É o preceptor que recebe os estudantes, apresentando-os e vinculando-os à equipe e ao serviço.

"O preceptor é a pessoa que dá a tua vida na Unidade de Saúde, é ele que faz a tua ligação com a equipe, $e$ considerando que o sistema funciona em equipe, o preceptor tem que te inserir na equipe." (Estudante 4)

Johns ${ }^{19}$ ao analisar o papel do preceptor na área da enfermagem, mostrou que uma das atribuições do preceptor no estágio curricular da graduação é fazer a conexão do estudante com sistema de saúde no qual ele está inserido, integrando-o ao cotidiano da profissão. Obtendo esse vínculo, há a possibilidade do estudante adquirir conhecimentos sobre como trabalhar em uma equipe multiprofissional.

Evidências na literatura têm identificado a receptividade como uma característica positiva da preceptoria $^{20}$ e mostrado que a experiência do trabalho em equipe multiprofissional de saúde, facilitada pelo preceptor, caracteriza-se um dos ganhos trazidos pelo estágio curricular ao estudante de graduação $^{10,11}$.

Para além da atuação em equipe multiprofissional, a vivência em um cenário de prática que possibilite o trabalho interdisciplinar, onde os profissionais de diferentes áreas trabalham juntamente em prol de um fim específico, unindo seus conhecimentos, também deveria fazer parte da formação de futuros cirurgiões-dentistas.

Estudo de Bispo, Tavares e Tomaz ${ }^{21}$ sobre a atuação dos preceptores de Unidades de Saúde da Família quanto à interdisciplinaridade mostrou, no entanto, desconhecimento na área e que os preceptores responsáveis por ensinar o estudante a trabalhar a partir da interdisciplinaridade, não se sentem preparados para passar esses 
conhecimentos. Os autores sugeriram a construção de um processo de educação permanente no âmbito interdisciplinar juntamente com as Instituições de Ensino Superior para essa área.

\section{Preceptor-professor: a importância da competência didático-pedagógica}

Outra característica fundamental para a atuação do preceptor é seu perfil para a adequada formação profissional, destacandose a competência didático-pedagógica para o ensino na saúde. Estudantes e preceptores assim se manifestaram:

"[...] se tu tem um preceptor mais ligado a essa parte didática, de educar e gostar dessa parte, que nem a minha estava sempre preocupada, então acho que faz a diferença." (Estudante 1)

"[...] o profissional tem que ter um mínimo de perfil de ensino, de educação, de pensar a formação, não só o trabalho." (Preceptor 3)

Revisão de literatura realizada por Mills, Francis e Bonner ${ }^{18}$ encontrou como requisito fundamental para a preceptoria a existência de uma capacidade didática do trabalhador, estabelecendo um ensino a partir do compartilhamento de experiências, ampliando a competência clínica e o desenvolvimento profissional do estudante dentro do ambiente de trabalho real.

"[...] o 'posto' é mais a vida real, [...] , o preceptor, claro, estava sempre lá pra me auxiliar, [...]ele me deixava mais preparada, eu me sentia mais responsável." (Estudante 10)

Epstein e Carlin ${ }^{22}$, ao estudarem as preocupações éticas na relação de preceptores e estudantes de enfermagem, relataram que a falta de apoio e segurança para o estudante na relação com seu preceptor gera um sentimento de ansiedade e faz com que o estudante sintase excluído do papel ativo no seu estágio, dificultando o aprendizado integrado no estágio curricular.

Ao ter uma postura para o ensino na saúde, o preceptor pode influenciar o estudante também em seu pensamento crítico, conversando com o estudante (competência da comunicação do preceptor), discutindo diagnósticos e procedimentos clínicos ou de funcionamento do serviço. O preceptor passa, assim, a ser um incentivador de uma prática reflexiva, dando segurança ao estudante nas atividades do estágio.

"[...] acho importante, sim, um preceptor que principalmente debate os assuntos contigo, tanto a parte clínica quanto a parte do funcionamento mesmo." (Estudante 7)

"[...] procuro conversar, [...] a gente, na própria atividade, vai conversando, isso é interessante, a gente está fazendo ali e já está tendo um feedback, uma reflexão." (Preceptor 6)

Duffy $^{23}$ estudou, por meio de uma pesquisa de abordagem qualitativa, a experiência dos preceptores na enfermagem que estimulavam a prática reflexiva nos estudantes que os acompanhavam. Mostrou que essa prática leva a uma melhor compreensão da experiência, desenvolvendo confiança e desafiando o aprendizado do estudante, criando ainda mais vínculo na relação com o preceptor, o que também foi obervado neste estudo.

\section{Integração teórico-prática: o preceptor enquanto facilitador da integração dos conhecimentos teóricos com a realidade do serviço}

Incentivando o pensamento reflexivo, o preceptor facilita ainda mais a aproximação 
do estudante com o trabalho realizado na APS, possibilitando que o mesmo associe os conhecimentos teóricos adquiridos na universidade com a realidade na qual está inserido.

"Os preceptores têm um papel muito importante para ajudar os alunos a vivenciarem plenamente o funcionamento da atenção básica, além de nos darem a oportunidade de debater assuntos que surgem durante o estágio, nos apresentando a visão do trabalhador do serviço." (Estudante 9)

Löfmark et al..$^{24}$, ao estudarem a satisfação dos estudantes com a preceptoria na enfermagem, relatou que a integração da teoria com a realidade de atendimentos era a responsável direta no aprendizado significativo do estudante, e que essa ligação era facilitada pelo estágio no serviço, bem como pela intervenção do preceptor no pensamento das atividades realizadas diariamente.

A integração da teoria com a prática no contexto da APS fica mais evidente para o estudante pelo fato dele estar trabalhando diretamente com alguém que vivencia essa realidade, um 'colega', não estando mais em contato somente com professores universitários e sim com trabalhadores do SUS. Essa situação de transição entre a formação acadêmica e o mundo do trabalho foi observada pelos estudantes.

“[...] o preceptor foi fundamental para a minha formação. Como ele sempre disse, foi um colega. Sabe, ele não tratava assim com uma visão de cima para baixo, ele sempre me tratou como igual e bem como um colega. Acho que foi fundamental ter bons colegas para o aprendizado." (Estudante 10)

$\mathrm{Da}$ mesma forma, professores $\mathrm{e}$ preceptores destacaram a relação diferenciada de ensino-aprendizagem entre os estudantes e os preceptores, quando comparada com a que os estudantes mantem com seus professores da universidade.

"No início, eles [os estagiários] chegam como alunos, eu lembro que um estudante até conversou comigo sobre isso: 'eu demorei umas duas semanas para entender que a preceptora não era minha professora, que ela era minha colega, e ela me tratava como colega', então eu acho que um dos maiores ganhos que o aluno teve foi essa relação com colega, que era uma coisa que ele não tinha na universidade, [...]." (Professor 3)

"[...] embora o preceptor esteja junto, não é o professor, é um colega, [o estagiário] sabe que tem um colega ali para ajudar, mas também não é a mesma coisa que é ter um professor [...]; sai dessa coisa do acadêmico para o profissional." (Preceptor 3)

A relação com o preceptor mostrou-se menos hierárquica e mais horizontal do que com o professor. Ao se sentir atuando com um 'colega de trabalho', o estudante tem mais autonomia e sente-se mais seguro, o que é refletido na transição da postura de estudante para a de um profissional.

Para o preceptor, a vivência com o estagiário trouxe uma experiência de integração cooperativa de conhecimentos, gerando uma constante renovação no cuidado em saúde.

"[...] o aluno é muito provocador, as perguntas deles são coisas que às vezes, para nós, passa batido, que a gente não tinha se dado conta, faz com que a gente tenha que estudar [...]”. (Preceptor 4) "[...] tem ganho para o preceptor, das 
coisas que eles [os estagiários] trazem da universidade, tanto das questões técnicas que a gente discute [...], quanto pela participação nos grupos, participação nas visitas, nas reuniões de equipe, [...] discussão de casos, a presença deles é importante, fortalece $o$ núcleo, e geralmente são alunos qualificados, então é bem importante." (Preceptor 5)

Estudo de Shannon et $a l .^{25}$, que analisou a opinião de diferentes profissionais da saúde de clínicas rurais na Austrália sobre a preceptoria que realizavam, da mesma forma, identificou que a troca de informações e o constante debate com os estudantes foi considerado um ponto positivo da preceptoria, gerando mais interesse para o trabalhador em se atualizar, buscando evidências científicas para sua prática profissional.

Essa troca de conhecimentos não fica restrita ao campo do estágio, mas também é percebida quando há a aproximação do serviço com a universidade. No curso de Odontologia da UFRGS foram criados espaços de trocas entre os preceptores, professores e estudantes, por meio de seminários teóricos e nas apresentações dos relatórios finais de estágio. Essa comunicação direta entre os protagonistas do estágio abre possibilidade para o preceptor participar ainda mais da formação do estudante juntamente com a instituição de ensino.

"A preceptoria não é um processo tão solitário, no início parecia que estava um pouco desconectado, a gente lá e os alunos e professores aqui, acho que agora a gente se sente muito mais integrado, mais participativo do processo, os seminários que têm, enfim, [...] não somos só coadjuvantes." (Preceptor 3)
A literatura mostra que um dos problemas relatados pelos trabalhadores ao exercerem a preceptoria é a falta de suporte das instituições de ensino ${ }^{26,27}$, o que não foi observado no relato dos preceptores no presente estudo. Tal fato pode ser explicado pela presença de um plano de trabalho adequado à demanda tanto dos trabalhadores quanto da universidade, tendo um planejamento prévio à inserção do estudante no serviço, o que traz benefícios à dinâmica das atividades ${ }^{2}$.

Os aspectos relatados no presente estudo possibilitam que o preceptor cumpra seu papel importante na efetividade de um estágio curricular no serviço de saúde. Experiências positivas de preceptoria, junto a um profissional considerado 'competente', resultam em um modelo de trabalhador para os estudantes ${ }^{22,27}$.

"Eu tenho muita admiração pela minha preceptora, eu sempre falo que eu quero ser uma profissional como ela é, porque é um exemplo de ética, conduta profissional, conduta clínica, assim, exemplar. Ela se doa, [...] busca sempre estar melhorando e ela também tem muita iniciativa, está sempre tendo ideias, tentando mudar [...] e criando coisas novas." (Estudante 11)

Ao final dessa etapa, o estudante que trabalhou junto a um preceptor que conseguiu inseri-lo e integrá-lo na equipe de saúde da APS, caracterizando-se pela receptividade ao estudante, pela competência didáticopedagógica e pela segurança na prática clínica, vivencia uma experiência privilegiada de ensino-aprendizagem. Tal experiência amplia os conhecimentos no campo da saúde e gera oportunidades para a formação de profissionais aptos para a atuação no SUS.

“[...] sem as parcerias, sem a 
aproximação com o serviço e com os preceptores, a proposta curricular do estágio não avança, e isso é muito importante [...] O que parece impactar mais durante o estágio é o que os estudantes conseguem ver na prática, convivendo com a equipe e com o preceptor, nas conversas, nesse cuidado diferenciado." (Professor 3).

\section{CONSIDERAÇÕES FINAIS}

O preceptor cirurgião-dentista da APS foi percebido como o profissional que integra o estudante à equipe de saúde e à rotina do serviço durante o período do estágio curricular. Quando demonstrou receptividade ao estudante inserindo-o no contexto do trabalho em equipe, competência didáticopedagógica e de comunicação, além de segurança no trabalho, atuou como facilitador da aprendizagem. Experiências positivas de preceptoria, junto a um profissional considerado 'competente', resultaram em um modelo de trabalhador e de colega para os estudantes.

A aproximação da universidade ao serviço e ao preceptor, apoiando-o nas atividades que desenvolve com os estudantes no cotidiano do trabalho na APS foi considerada importante para a atuação desse preceptor, devendo ser entendida como condição necessária para a integração ensinoserviço-comunidade.

A temática pesquisada não se esgota com os resultados desta pesquisa qualitativa exploratória, mas sim, abre oportunidade para a realização de outros estudos com abordagem qualitativa em diferentes Instituições de Ensino Superior no país que contribuam com a compreensão da atuação do preceptor na formação em saúde.

\section{ABSTRACT \\ Perceptions about the preceptor Dental Practitioner of the Primary Health Care in Dental education}

The implementation of the National Curricular Guidelines for the healthcare area in Brazil and in particular, for dentistry, brought the need to integrate teaching with the Unified Health System. This integration is enabled by the experience of the students in the curricular internships within the public health service, accompanied by the preceptor, a professional who works in the service. The study aims to comprehend the perceptions of the dental practitioners, supervisor teachers and students about the preceptors of the Dentistry curricular internship in the Primary Health Care (PHC). A qualitative exploratory study was conducted through semi structured interviews with students, teachers and preceptors $(n=20)$ linked to the graduation curricular internship of the Dental Course of the Universidade Federal do Rio Grande do Sul in the PHC services. The preceptor dental practitioner was perceived as the professional that inserts and integrates the student in the health team and the routine of the service during the internship. When he demonstrated receptivity to the student, didacticpedagogical competence and security at work, acted as a learning facilitator. Positive experiences of preceptorship, along with a professional considered "competent", resulted in a worker model and a colleague to the students. The student's relation with the preceptor was not perceived as the student's relation with the teacher in the university. The approach of the university and the preceptor, supporting him in the activities that he develops with the student in the work routine must be understood as a necessary condition for the integration teaching-servicecommunity.

Descriptors: Dental Education. Primary Health Care. Health Services. Preceptor. Qualitative Research. 


\section{REFERÊNCIAS}

1. Reis SMAS, Oliveira AG, Gonçalves LC, Costa MM, Oliveira JEC, Costa CRRC, et al. Formação odontológica: persiste o descompasso entre o perfil de cirurgiãodentista atualmente formado $\mathrm{e}$ as demandas da sociedade por saúde bucal. Rev Ed Popular. 2009;8(1):86-97.

2. Souza AL, Carcereri DL.Estudo qualitativo da integração ensino serviço em um curso de graduação em Odontologia. Interface (Botucatu). 2011;15(39):1071-84.

3. Resolução CNE/CES 3/2002, de 19 de Fevereiro de 2002. Institui Diretrizes Curriculares Nacionais do Curso de Graduação em Odontologia. Diário Oficial da União, 4 Mar 2002. Disponível em: http://portal.mec.gov.br/cne/arqui vos/pdf/CES032002.pdf

4. Morita MC, Kriger L. Mudanças nos cursos de Odontologia e sua interação com o SUS. Rev ABENO. 2004;4(1):17-21.

5. Moysés ST, Kriger L, Moysés SJ. Saúde bucal das famílias: trabalhando com evidências. São Paulo: Artes Médicas; 2008.

6. Toassi RFC, Davoglio RS, Lemos VMA. Integração ensino-serviço-comunidade: $\mathrm{o}$ estágio na atenção básica da graduação em Odontologia. Educ Rev. 2012;28 (4):223-42.

7. Ministério da Saúde. Secretaria de gestão do Trabalho e da Educação na Saúde. Glossário Temático Gestão do Trabalho e da Educação na Saúde. Brasília; 2009. Disponível em: http://bvsms.saude.gov. br/bvs/publicacoes/glossario_sgtes.pdf

8. Botti SHO, Rego S. Preceptor, supervisor, tutor e mentor: quais são seus papéis? Rev Bras Educ Med. 2008; 32(3):363-73.
9. Universidade Federal do Rio Grande do Sul. Faculdade de Odontologia. Projeto Político Pedagógico. Porto Alegre; 2005 [acesso em 9 Jul 2014]. Disponível em: http://www.ufrgs.br/odonto/projeto_peda gogico_Odontologia_curso_diurno

10. Toassi, RFC, Baumgarten A, Warmling CM, Rossoni E, Rosa, AR, Slavutzky SMB. O ensino nos serviços de Atenção Primária à Saúde do Sistema Único de Saúde (SUS) na formação de profissionais de saúde no Brasil. Interface (Botucatu). 2013;17(45):385-92.

11. Barreto VHL, Monteiro ROS, Magalhães GSG, Almeida RCC, Souza LN. Papel do preceptor da Atenção Primária à Saúde em saúde da formação da graduação e pós-graduação da Universidade Federal de Pernambuco - um termo de referência. Rev Bras Educ Méd. 2011;35(4):578-83.

12. Tong A, Sainsbury P, Craig J. Consolidated criteria for reporting qualitative research (COREQ): a 32-item checklist for interviews and focus groups. Int J Qual Health Care. 2007;19(6):34957.

13. Turato ER. Tratado de metodologia da pesquisa clínico-qualitativa: construção teórico-epistemológica, discussão comparada e aplicação nas áreas da saúde e humanas. 3a ed. Petrópolis, RJ: Vozes; 2008.

14. Bardin L. Análise de conteúdo. São Paulo: Edições 70; 2011.

15. Werneck MAF, Senna MIB, Drumond MM, Lucas SD. Nem tudo é estágio: contribuições para o debate. Ciên Saúde Coletiva. 2010;15(1):221-31.

16. Hood JG. Service learning in dental education: meeting needs and challenges. J Dent Educ. 2009;73(4) 454-63.

17. Yoder KMA. Framework for service- 
learning in dental education. J Dent Educ. 2006;70(2):115-23.

18. Mills JE, Francis KL, Bonner A. Mentoring, clinical supervision and preceptoring: clarifying the conceptual definitions for Australian rural nurses. A review of the literature. Rural Remote Health. 2005;5(3):410-19.

19. Johns C. Depending on the intent and emphasis of the supervisor, clinical supervision can be a different experience. J Nurs Manag. 2001;9(3):139-45.

20. Broadbend M, Moxham L, Sander T, Walker S, Dwyer T. Supporting bachelor of nursing students within the clinical environment: perspectives of preceptors. Nurse Educ Pract. 2014;14(4):403-40.

21. Bispo EPF, Tavares CHF, Tomaz JMT. Interdisciplinaridade no ensino em saúde: o olhar do preceptor na Saúde da Família. Interface (Botucatu). 2014;18 (49):337-50.

22. Epstein I, Carlin K. Ethical concerns in the student/preceptor relationship: a need for change. Nurse Educ Today. 2012;32(8):897-902.

23. Duffy A. Guiding students through reflective practice - the preceptors experiences. A qualitative descriptive study. Nurse Educ Pract. 2009;9(3):16675 .
24. Löfmark A, Thorkildsen K, Raholm MB, Natvig GK. Nursing students' satisfaction with supervision from preceptors and teachers during clinical practice. Nurse Educ Pract. 2012;12 (3):164-9.

25. Shannon SJ, Walker-Jeffreys $M$, Newbury JW, Cayetano T, Brown K, Petkov J. Rural clinician opinion on being a preceptor. Rural Remote Health. 2006;6(1):490-503.

26. Bourbonnais FF, Kerr E. Preceptoring a student in the final clinical placement: reflections from nurses in a Canadian Hospital. J Clin Nurs. 2007;16(8):1543-9.

27. Hallin K, Danielson E. Preceptoring nursing students: registered Nurses' perceptions of nursing students' preparation and study approaches in clinical education. Nurse Educ Today. 2010; 30(4): 269-302.

Correspondência para:

Ramona Fernanda Ceriotti Toassi e-mail: ramona.fernanda@ufrgs.br Faculdade de Odontologia da UFRGS Rua Ramiro Barcelos 2492, 2o andar 90035-003 Porto Alegre, RS 